\title{
Ganciclovir Treatment in Infants With Cytomegalovirus Infection and Cholestasis
}

\author{
Björn Fischler, Thomas H. Casswall, Petter Malmborg, and Antal Nemeth \\ Department of Pediatrics, Huddinge University Hospital, Karolinska Institutet, Stockholm, Sweden
}

\section{ABSTRACT}

Background: The authors have previously described an association between cytomegalovirus (CMV) infection and intrahepatic and extrahepatic forms of neonatal cholestasis. Pediatric use of the antiviral drug ganciclovir to treat patients with CMV infection has increased. In this study, infants with CMV infection and cholestasis were treated with ganciclovir.

Methods: Six infants with cholestasis (age, 3-16 weeks) and with signs of ongoing CMV infection were treated with intravenous ganciclovir for 3 to 7 weeks and observed for 4 to 31 months after treatment. Two patients had biliary atresia, one had suspected septo-optic dysplasia and three had no obvious cause for intrahepatic cholestasis other than ongoing CMV infection.
\end{abstract}

Results: Four patients, including one with biliary atresia, re- sponded to the treatment, whereas two patients, including the one with septo-optic dysplasia did not. The latter patient had episodes of symptomatic hypoglycemia during the treatment, which was subsequently stopped. Liver function at the end of follow-up was good in four patients, intermediate in one, and poor in one.

Conclusion: Ganciclovir treatment may be beneficial in infants with CMV-associated intrahepatic cholestasis, but controlled studies are needed. Because of the possible side effect of hypoglycemia, infants with cholestasis who have increased risk for such episodes should not be treated. JPGN 34:154-157, 2002. Key Words: Neonatal cholestasis-Extrahepatic biliary atresia-Cytomegalovirus-Ganciclovir-Treatment. (c) 2002 Lippincott Williams \& Wilkins, Inc.

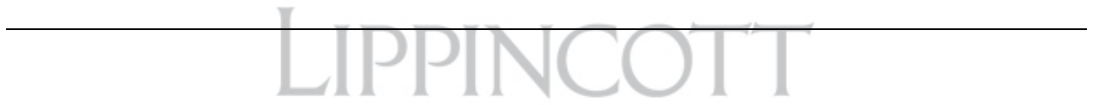

The list of causes for neonatal cholestasis includes biliary atresia (BA) and a number of intrahepatic problems such as infections, metabolic conditions, and chronic familial cholestatic diseases (1). In serologic studies, various viral infections have been associated with intrahepatic forms of neonatal cholestasis (1). Cytomegalovirus (CMV) is the virus most commonly implicated, and CMV antigen has been detected in liver biopsy specimens from these patients (2).

Several viral infections have been implicated in causing BA (3-6). We have found an association between CMV infection and BA (7). Serologic signs of ongoing CMV infection were found in 8 of 21 patients with BA as compared with 2 of 35 age-matched controls $(P<$ $0.01)$. Furthermore, polymerase chain reaction (PCR) detected CMV-DNA in liver biopsy specimens from 9 of 18 patients with BA.

The possible link between CMV infection and both intrahepatic and extrahepatic forms of neonatal cholestasis raises the issue of antiviral therapy. Ganciclovir has

Received January 29, 2001; accepted September 4, 2001.

Address correspondence and reprint requests to Dr. Björn Fischler, Department of Pediatrics, Huddinge University Hospital, Karolinska Institutet, SE-141 86 Huddinge, Sweden (e-mail: bjorn.fischler@ pediat.hs.sll.se). been studied extensively in the treatment of adult CMV infection, and increasing evidence supports its use in immunosuppressed children (8). However, limited data are available on the use of ganciclovir in young infants. Most of the information is derived from studies of patients with congenital CMV infection and mainly central nervous system (CNS) symptoms $(9,10)$, and not from patients in whom the infection primarily affects the liver. Thus, the aim of this report was to retrospectively describe the effect of ganciclovir in a selected group of infants with $\mathrm{CMV}$-associated cholestasis.

\section{METHODS}

\section{Patients}

Six infants with CMV infection and cholestasis (patients $1-6$, Table 1) who were seen at our tertiary center for pediatric hepatology were included in this study. All patients had clinical and biochemical signs of cholestasis and were younger than 4 months of age. Ongoing CMV infection was defined by the detection of CMV-IgM in serum or of CMV in the urine. Two patients had BA (patients 1 and 2); one had neonatal hypoglycemia and optic nerve hypoplasia, suggesting septo-optic dysplasia (patient 3); and three had intrahepatic cholestasis with no other metabolic or infectious cause other than CMV infection 
TABLE 1. Clinical and virologic data on $6 C M V$-infected cholestatic patients treated with gancyclovir

\begin{tabular}{ccccccccc}
\hline $\begin{array}{c}\text { Patient } \\
\text { no. }\end{array}$ & Sex & Diagnosis & $\begin{array}{c}\text { CMV-DNA } \\
\text { Guthrie card* }\end{array}$ & CMV-IgM & $\begin{array}{c}\text { CMV } \\
\text { urine }\end{array}$ & $\begin{array}{c}\text { Maternal } \\
\text { CMV }\end{array}$ & $\begin{array}{c}\text { Age at treatment } \\
\text { (wk) }\end{array}$ & $\begin{array}{c}\text { Treatment duration } \\
(\text { wk) }\end{array}$ \\
\hline 1 & F & BA & NP & - & + & IgG+/IgG++ & 3,9 & 7 \\
2 & F & BA & NP & + & NP & IgG+/IgG++ & $4,2 \dagger$ \\
3 & F & SOD? & - & - & + & IgG+/gG+ & 10 & 4 \\
4 & M & NS & - & + & + & IGg+/IgG+ & 11 & 7 \\
5 & M & NS & + & + & - & NP/IgG+ & 12 & 4 \\
6 & M & NS & NP & - & + & IgG+/IgG++ & 16 & \\
\hline
\end{tabular}

* Sampled and stored at approximately 3 days of age.

$\dagger$ Patient was treated twice.

CMV, cytomegalovirus; IgM, immunoglobulin M; BA, biliary atresia; SOD?, suspected septo-optic dysplasia; NS, nonspecific intrahepatic cholestasis;NP, not performed; +, positive; -, negative; IgG+/IgG++, significant increase in immunoglobulin $\mathrm{G}$ levels in samples from the first trimester of pregnancy and from the time of the infant's examination.

(patients 4-6). Liver biopsy, which was performed at the time of first investigation, was compatible with BA in patients 1 and 2 , and showed signs of giant-cell hepatitis in patients 3 through 6. No CMV inclusions were seen, and neither immunohistochemistry nor in situ hybridization for CMV was performed.

In patient $1, \mathrm{CMV}$ infection was diagnosed before 3 weeks of age, suggesting congenital infection (11). In the other five patients, CMV infection was diagnosed after 3 weeks of age. Hence it was impossible to differentiate among prenatal, perinatal, or postnatal infection. However, for three of those five patients, retrospective CMV-DNA analysis by PCR on the Guthrie card, sampled at 3 days of age to detect inborn errors of metabolism, was performed (12). One of the patients tested positive, suggesting viremia at birth, whereas two tested negative (Table 1).

Maternal sera, from the time of the first investigation of the infants were analyzed in all cases. Stored maternal sera, from the first trimester of the pregnancy were available in all but one case. Primary CMV infection, as defined by the detection of CMV-IgM in the serum was not found in any of the mothers. However, for three of five mothers with paired sera available, the CMV-IgG levels significantly increased between the two tests, suggesting reactivation of the CMV infection (Table 1).

\section{Treatment}

All infants with cholestasis were treated with intravenous ganciclovir for 3 to 7 weeks. Patient 1 was treated twice, before and after portoenterostomy (Kasai procedure). The initial dosage was $5 \mathrm{mg} / \mathrm{kg}$ body weight twice daily for all patients. After 2 weeks of treatment, the dosage was reduced to $5 \mathrm{mg} / \mathrm{kg}$ once daily in all patients except for patient 3 , for whom the dose was not changed. Mean follow-up time after ganciclovir treatment was 14.5 months (range, 4-31 months).

All patients received standard supportive treatment for cholestasis, including supplementation with fat-soluble vitamins and ursodeoxycholic acid (UDCA). Treatment with UDCA was started 2 to 6 weeks before ganciclovir was started in patients 4,5 , and 6 . In these patients, ganciclovir was added because of continuous cholestatic liver disease. In patients 1 and 2, the two drugs were started at the same time, whereas in patient 3, antiviral treatment preceded UDCA treatment. Patients 1 and 2 underwent portoenterostomy.

\section{Outcome Variables}

Biochemical response to the treatment was defined as a marked decrease in serum levels of the cholestatic markers bilirubin and fasting bile acids. Patients in whom CMV-DNA could not be detected by PCR in leukocytes or in whom CMV could not be isolated in the urine after treatment were considered to respond virologically. The clinical outcome at last follow-up was considered 1) good, with no or only minor liver disease; 2) intermediate, with chronic compensated liver disease; or 3) poor, if the patient underwent liver transplantation or died.

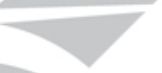

Methods

Cytomegalovirus IgG and IgM were detected in serum using in-house enzyme linked immunoassays (ELISA) $(13,14), \mathrm{CMV}$ was isolated in the urine using routine methods (7), and CMVDNA was detected in leukocytes by PCR (15). Cytomegalovirus DNA was detected in stored Guthrie cards as previously described (12). Serum levels of alanine aminotransferase, aspartate aminotransferase, $\gamma$-glutamyl transpeptidase, bilirubin, and fasting bile acids, and blood levels of hemoglobin, white blood cells, and platelets were analyzed according to hospital routines.

\section{Ethical Considerations}

The local ethics committee at Huddinge University Hospital approved all virologic examinations. Parents gave informed consent for treatment with ganciclovir.

\section{RESULTS}

Four patients (patients 1, 4, 5, and 6) responded biochemically to the treatment, and four patients (patients 1 , 2, 4, 6 responded virologically (Table 2). Patient 5 had not responded virologically when tested after treatment, but had no detectable CMV-DNA in leukocytes at follow-up 1 year later. Liver failure developed in patient 2 shortly after the portoenterostomy procedure, and the patient died of surgical complications after liver transplantation at 6 months of age, that is, 3 months after the antiviral treatment. The patient with suspected septooptic dysplasia (patient 3) had episodes of symptomatic hypoglycemia during ganciclovir treatment, which was subsequently withdrawn. At follow-up, no signs of liver disease were detected and no further episodes of hypogly- 
TABLE 2. Clinical, biochemical, and virological results of gancyclovir treatment in 6 CMV-infected cholestatic infants

\begin{tabular}{|c|c|c|c|c|c|c|c|c|c|c|c|}
\hline \multirow{2}{*}{$\begin{array}{c}\text { Patient } \\
\text { no. }\end{array}$} & \multirow{2}{*}{$\begin{array}{l}\text { Follow-up } \\
\text { time (mo) }\end{array}$} & \multicolumn{3}{|c|}{$\begin{array}{l}\text { S-bilirubin } \\
(\mu \mathrm{mol} / \mathrm{L})\end{array}$} & \multicolumn{3}{|c|}{$\begin{array}{l}\text { S-bile acids } \\
(\mu \mathrm{mol} / \mathrm{L})\end{array}$} & \multicolumn{3}{|c|}{ CMV-DNA* } & \multirow[b]{2}{*}{ Outcome } \\
\hline & & BT & AT & LT & BT & AT & LT & BT & AT & LT & \\
\hline 1 & 31 & 131 & 40 & 4 & 156 & 77 & 45 & NP & - & - & II \\
\hline 2 & 4 & 237 & 169 & 373 & 214 & NP & 375 & NP & - & NP & III \\
\hline 3 & 12 & 99 & 70 & 4 & NP & 370 & 17 & $\mathrm{NP}_{\dagger}$ & - & - & I \\
\hline 4 & 12 & 147 & 7 & 5 & 134 & 20 & 4 & NP & NP\$ & NP & I \\
\hline 5 & 12 & 117 & 26 & 7 & 315 & 169 & 1 & NP & + & - & I \\
\hline 6 & 17 & 14 & 7 & 8 & 183 & 12 & NP & NP & - & NP & I \\
\hline
\end{tabular}

* Detected in leukocytes by polymerase chain reaction.

$\dagger$ Positive CMV-DNA in serum (polymerase chain reaction).

\$ Negative CMV isolation in the urine.

CMV, cytomegalovirus; BT, before treatment; AT, immediately after treatment; LT, long-term follow-up; NP, not performed; +, positive; -, negative; I, none or minor liver disease; II, chronic compensated liver disease; III, liver transplanted and/or dead.

cemia were reported. However, the patient received substitution therapy for hypothyroid disease beginning at the age of 14 months. Apart from the episode of hypoglycemia in this patient, no other clinical or biochemical side effects occurred that required withdrawal of ganciclovir treatment. The outcome at the end of follow-up was good in four patients (patients 3-6), intermediate in one (patient 1), and poor in one (patient 2).

\section{DISCUSSION}

The previously found association between CMV infection and various forms of neonatal cholestasis can be interpreted in various ways: CMV can be 1) a causative agent, 2) a factor not initiating but aggravating the cholestatic process, or 3) an "innocent bystander." Antiviral treatment may be relevant in the first and second interpretation, but not in the third. Considering the morbidity and mortality for selected patients with CMV-associated neonatal cholestasis, a pilot study with ganciclovir treatment was justified.

The results from this study suggest that antiviral treatment is valuable for infants with no obvious cause for intrahepatic cholestasis other than CMV infection. Thus, cholestatic markers for patients 4, 5, and 6 improved significantly, and their long-term prognosis was good. Furthermore, no significant clinical or biochemical side effects were noted in these three patients. The treatment effect in this group of patients may simply reflect the natural history of the disorder (16). However, the outcome in infants with CMV-associated cholestasis is not always benign $(17,18)$.

All patients were treated with UDCA, an established treatment in infants with cholestasis (1). In patients 4, 5, and 6, cholestasis did not improve with UDCA alone, thus ganciclovir was added. That this coincided with biochemical improvement suggests that adding ganciclovir was significant.

For the small group of patients with BA, the effect of treatment was less clear. Improvement in cholestatic markers in patient 1 may be attributed partially to ganciclovir treatment, but UDCA also may have affected outcome. However, the most important cause for improvement was probably a successful Kasai procedure. Conversely, the lack of improvement in patient 2 was probably not because of failure or of any side effect of antiviral treatment, but because the patient belonged to the group that underwent unsuccessful portoenterostomy procedures.

In patient 3, no immediate improvement in cholestasis was seen during treatment. Episodes of symptomatic hypoglycemia were probably a side effect of treatment; no more such episodes were seen after cessation of treatment. The World Health Organization database on adverse drug reactions reports a few cases of hypoglycemia in patients treated with ganciclovir (19). Considering the possibility of a midline defect in our patient, ganciclovir treatment may have triggered hypoglycemic episodes in a predisposed individual.

The dosage used in this pilot study was based on previous studies of CMV treatment in neonates, with symptoms predominantly from the CNS $(9,10,20)$, and data on the treatment children and adults who underwent transplantation (8). Prolonged treatment of 3 months may yield better virologic and perhaps also clinical and biochemical results, as suggested by Nigro et al. (21). However, this poses other problems, because the patient will probably require a central line catheter for a longer period, which is inconvenient and increases the risk of systemic infections.

Because of the retrospective nature of the study, the virologic follow-up data are not complete for all patients. However, we think that the available virologic data give valuable information. Thus, in patients $1,3,4$, and 6 , the virologic results after treatment seemed to correlate to a biochemical response. In patient 5 , the biochemical response seemed to precede virologic response. The reason for this discrepancy is unclear, but the data may strengthen the case for prolonged antiviral treatment (21). 
Primary infection was not detected in any mother, although it could not be ruled out in the mother of patient 5. The increase in CMV-IgG levels in three of five mothers analyzed suggests reactivation of the infection. This does not necessarily imply that the infants of those three mothers were viremic at birth (12).

Patients 1 (mother had increased CMV-IgG) and 5 (no prenatal serum available) were considered to have congenital infection, and patients 3 and 4 (both mothers had steady CMV-IgG levels) not to have congential infection. For patients 2 and 6 , insufficient data were available.

Because most infants with cholestasis are investigated at the age of 4 to 8 weeks, a connection cannot be made among prenatal, perinatal, and postnatal CMV infections $(7,11)$. A prenatal/congenital infection may be more serious and therefore more important to treat. However, treating a perinatal or early postnatal infection may be more appropriate because the virus may cause less permanent damage. Although our data are incomplete on this issue, we noticed that the two patients with congenital infection (patient 1 and 5) did respond biochemically. At follow-up, they also had responded virologically.

In a recent report on ganciclovir treatment in infants with congenital CMV infection, no difference in the outcome of liver disease was seen in treated patients compared with untreated patients (22). However, treatment seemed beneficial in light of CNS symptoms. The organ specificity of the CMV infection may explain this difference, but it also may be because of ganciclovir treatment.

Considering the potential number of infants with CMV-associated cholestasis, the availability of the antiviral drug ganciclovir, and the results of this pilot study, we suggest that a larger, controlled study is necessary. Such a study would be important in separating the effects of ganciclovir from those of UDCA or other choleretics and in evaluating whether CMV is of true pathogenetic importance in these patients. Furthermore, adding CMVDNA analysis by PCR on stored Guthrie cards from these patients may provide a useful tool for predicting the outcome of the treatment.

\section{REFERENCES}

1. Dellert SF, Balistreri WF. Neonatal cholestasis. In: Walker A, Durie P, Hamilton J, Walker-Smith J, eds. Pediatric Gastroenterology. 3rd ed. Lewiston, NY: BC Decker: 2000:880-94.

2. Kage M, Kosai KI, Kojiro M, et al. Infantile cholestasis due to cytomegalovirus infection of the liver. Arch Pathol Lab Med 1993; 117:942-4.
3. Morecki R, Glaser J, Cho S, et al. Biliary atresia and reovirus type 3 infection. N Engl J Med 1982;307:481-4.

4. Tyler KL, Sokol RJ, Oberhaus SM, et al. Detection of reovirus RNA in hepatobiliary tissue from patients with extrahepatic biliary atresia and choledochal cysts. Hepatology 1998;27:1475-82.

5. Riepenhoff-Talty M, Gouvea V, Evans MJ, et al. Detection of group $\mathrm{C}$ rotavirus in infants with extrahepatic biliary atresia. $J$ Infect Dis 1996;174:8-15.

6. Drut R, Drut RM, Gomez MA, et al. Presence of human papillomavirus in extrahepatic biliary atresia. J Pediatr Gastroenterol Nutr 1998;27:530-5.

7. Fischler B, Ehrnst A, Forsgren M, et al. The viral association of neonatal cholestasis in Sweden, a possible link between cytomegalovirus and extrahepatic biliary atresia. $J$ Pediatr Gastroenterol Nutr 1998;27:57-64.

8. Crumpacker CS. Ganciclovir. N Engl J Med 1996;335:721-9.

9. Halwachs G, Kutcshera J, Tiran A, et al. Antiviral treatment of congenitally infected children with a positive polymerase chain reaction in the cerebrospinal fluid [Extended abstract]. Scand $J$ Infect Dis Suppl 1995;99:89-90.

10. Whitley RJ, Cloud G, Gruber W, et al. Ganciclovir treatment of symptomatic congenital cytomegalovirus infection-results of a phase II study. J Infect Dis 1997;175:1080-6.

11. Britt WJ, Alford CA. Cytomegalovirus. In: Fields BN, Knipe DM, Howöey PM, et al., eds. Fields Virology. 3rd ed. Philadelphia: Lippincott-Raven; 1996:2493-523.

12. Fischler B, Rodensjö P, Nemeth A, et al. CMV-DNA detection on Guthrie cards in patients with neonatal cholestasis. Arch Dis Child Fetal Neonatal Ed 1999;80:F130-4.

13. Sundkvist VA, Wahren B. An interchangeable ELISA for cytomegalovirus antigen and antibody. J Virol Methods 1981;2: 301-12.

14. Ahlfors K, Forsgren M, Griffiths P, et a;. Comparison of four serological tests for the detection of specific immunoglobulin $M$ in cord sera of infants congenitally infected with cytomegalovirus. Scand J Infect Dis 1987;19:303-8.

15. Ehrnst A, Barkholt L, Lewensohn-Fuchs I, et al. CMV PCR monitoring in leukocytes of transplant patients. Clin Diagn Virol 1995; 3:139-53

16. Mowat AP, Psacharopoulos HT, Williams R. Extrahepatic biliary atresia versus neonatal hepatitis. Review of 137 prospectively investigated infants. Arch Dis Child 1976;51:763-70.

17. Chang MH, Hsu HC, Lee CY, et al. Neonatal hepatitis: A followup study. J Pediatr Gastroenterol Nutr 1987;6:203-7.

18. Zuppan CW, Bui HD, Grill BG. Diffuse hepatic fibrosis in congenital cytomegalovirus infection. J Pediatr Gastroenterol Nutr 1986;5:489-91.

19. World Health Organization. Intdis (International Drug Information System): WHO's adverse drug reactions database. Available at: http://www.who.int/druginformation.

20. Attard-Monalto SP, English MC, Stimmler L, et al. Ganciclovir treatment of congenital cytomegalovirus infection: a report of two cases. Scand J Infect Dis 1993;25:385-8.

21. Nigro G, Krzystofiak A, Bartmann U, et al. Ganciclovir therapy for cytomegalovirus associated liver disease in immunocompetent or immunocompromised children. Arch Virol 1997;142:573-80.

22. Kimberlin DW, Lin CY, Sanchez P, et al. Ganciclovir (GCV) treatment of symptomatic congenital cytomegalovirus (CMV) infections: results of a phase III randomized trial. 40th Interscience Conference on Chemotherapy and Antimicrobials [abstract]. Antimicrob Agents Chemother 2000;44:1942. 